\title{
Rechnergestützte Entwicklungsumgebung zur Konstruktion von Tailored-Forming-Bauteilen
}

Tim Brockmöller ${ }^{1}$, Renan Siqueira ${ }^{1}$, Iryna Mozgova ${ }^{1}$, Roland Lachmayer $^{1}$

${ }^{1}$ Institute of Product Development and Engineering Design (IPeG), Leibniz University Hannover, Germany

\section{Abstract}

This article describes an approach for designing knowledge based Computer Aided Design (CAD) models to represent the complete solution space of the Tailored Forming process chain by depicting the production stages of a component. The aim is to use this knowledge base to determine during the product development process whether the desired component can be manufactured with the available equipment, even if the design engineer does not have the necessary expert knowledge. In addition to the model structure, the implementation of the manufacturing restrictions and the dependencies between the different production stages are shown. The procedure is described using the newly developed tailored forming process chain as an example.

Keywords: Tailored Forming, Computer Aided Design, Knowledge Based Design

\section{Einleitung}

Dieser Beitrag entstand im Rahmen des Sonderforschungsbereiches (SFB) 1153: „Prozesskette zur Herstellung hybrider Hochleistungsbauteile durch Tailored Forming". Bei dieser Fertigungstechnologie werden Bauteile mit lokal an- 
gepassten Eigenschaften hergestellt, indem für die jeweils bestehenden Einsatzbedingungen geeignete Werkstoffe verwendet werden. Zu Beginn werden zwei Monomaterialhalbzeuge stoffschlüssig gefügt, anschließend thermo-mechanisch umgeformt, wärmebehandelt und spanend nachgearbeitet (s. Bild 1). Die thermomechanische Umformung dient dabei zur Verbesserung des Stoffschlusse in der Fügezone. Durch die Verwendung verschiedener Werkstoffe, lassen sich die einzelnen Bereiche dieser hybriden Bauteile im Vergleich mit Monomaterialbauteilen wesentlich besser ausnutzen, was z.B. zu einer Gewichts- oder Kosteneinsparung führt. Anwendungsbereiche bieten sich besonders beim Bestreben des Downsizings von Motoren- und Fahrwerkskomponenten in der Automobilindustrie, bei denen im Einsatz eine hohe Effizienz gefordert ist und Monomaterialbauteile an ihre technologischen Grenzen stoßen [1].

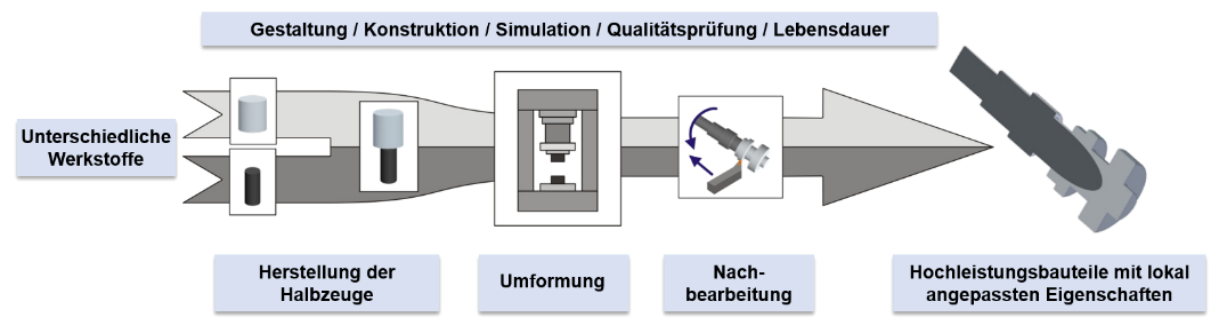

Bild 1: Schematische Darstellung der Tailored-Forming-Prozesskette, nach [2]

Da es sich bei der Tailored-Forming-Technologie um eine neue und komplexe Prozesskette handelt, ist bisher unbekannt, wie die Fertigungsmöglichkeiten und -restriktionen aussehen. Daher wird im Rahmen des Teilprojektes "Gestaltung und Dimensionierung" erforscht, wie das erarbeitete Wissen gespeichert und abgerufen werden kann. Dafür befindet sich eine rechnerunterstützte Entwicklungsumgebung im Aufbau, die Werkzeuge zur Potentialfindung, Wissensspeicherung [3] sowie zur Auslegung [4] und Konstruktion von Tailored-Forming-Bauteilen bereitstellt [5]. In diesem Artikel wird ein Ansatz für eine rechnerunterstützte Konstruktionsmethode beschrieben, mit dem die entwickelten Tailored-Forming-Bauteile fertigungsgerecht auf die zur Verfügung stehende Fertigungsprozesskette angepasst werden können. Dabei werden durch wissensbasierte CAD-Modelle relevante Fertigungsstufen abgebildet und miteinander verknüpft, sodass sich eine Änderung im Modell der finalen Bauteilgeometrie, auf die Modelle der Fertigungsstufen auswirkt. Eine Fertigungsstufe liegt in diesem Zusammenhang vor, wenn durch einen Fertigungsprozess die Geometrie des Bauteils verändert wird.

Die Ziele dabei sind zum einen bereits während der Produktentwicklung zu überprüfen, ob das Tailored-Forming-Bauteil mit den zur Verfügung stehenden 
Fertigungsmöglichkeiten hergestellt werden kann. Zum anderen werden die Bauteilgeometrien nach den einzelnen Fertigungsstufen abgebildet, um daraus z.B. die für die Herstellung benötigten Werkzeuge entwickeln zu können. Darüber hinaus soll es einem Konstruierenden möglich sein, Tailored-Forming-Bauteile innerhalb zugelassener Parameter der Prozesskette konstruieren zu können, ohne dabei zwangsläufig über das nötige Expertenwissen der Technologie zu verfügen. Die Herausforderungen dabei sind die in der Prozesskette zu berücksichtigen fertigungsstufenübergreifende Fertigungsrestriktionen in die Modelle zu implementieren. Außerdem müssen die Modelle in der Lage sein, sich parametrisch und topologisch bei Änderungen der finalen Bauteilgeometrie und über die Fertigungsstufen hinweg anpassen zu können. Dafür wird ein flexibler und robuster Modellaufbau benötigt.

Als Beispiel wird ein rotationssymmetrisches Demonstratorbauteil aus dem SFB verwendet. Die Verbindung der monomaterial-Halbzeuge wird durch einen Reibschweißprozess hergestellt. Das hybride Halbzeug wird anschließend durch Fließpressen thermomechanisch umgeformt, wärmebehandelt und spanend bearbeitet, um die Endgeometrie zu erstellen.

\section{Stand der Technik}

Da moderne CAD-Systeme einen parametrischen Modellaufbau unterstützen, ist es möglich Konstruktions- und Prozesswissen durch Regeln in die CADModelle zu implementieren [6]. Damit lassen sich diese wissensbasierten Modelle direkt in den Produktentwicklungsprozess einbinden, da bereits bewährte Lösungsprinzipien für spezifische Aufgaben zur Verfügung gestellt werden. Auf diese Weise lassen sich Routinearbeiten automatisieren und der Aufwand während des Produktentwicklungsprozesses wird minimiert [7]. Zu beachten ist dabei der erhöhte Aufwand der Modellplanung und -erstellung. Außerdem ist eine ständige Modellpflege notwendig, wenn neue Erkenntnisse berücksichtigt und die Modelle erweitert werden sollen [8].

Der Generative Design Approach (GDA), beinhaltet eine Modellierungsmethode, mit der wissensbasierte Modelle erstellt werden können, die besonders flexibel bei Änderungen oder Erweiterungen der Bauteilgeometrie sind. Die Idee dieser Modellierungsmethode ist es, die Anzahl der Abhängigkeiten zwischen den Modellparametern zu minimieren und eine Modellstruktur aufzubauen, die unabhängig von der Baustruktur des Bauteils ist. Das bedeutet, dass ein einzelnes Bauteil im CAD-System nicht mehr durch eine einzige Einzelteildatei dargestellt wird, sondern es sich um eine Baugruppendatei handelt. Die grundlegende Dimensionierung des Gesamtmodells wird durch ein übergeordnetes Skelett gesteuert, das die einzelnen Elemente des Modells miteinander 
verknüpft. Das Bauteil wird in verschiedene Gestaltungszonen eingeteilt, die ihrerseits jeweils durch eine Einzelteildatei dargestellt werden. Die Einzelteildateien werden als sogenannte Gestaltelemente bezeichnet und sind eine Gestaltlösung für die jeweilige Gestaltungszone. Die Geometrie der Gestaltelemente ist parametrisiert und lässt sich mittels einiger weniger Schnittstellenparameter durch übergeordneter Führungsparameter [6] manipulieren. Auf diese Weise wird z.B. ein stetiger Geometrieübergang zwischen den Gestaltelementen gewährleistet. Für jede Gestaltungszone werden verschiedene Gestaltelemente mit unterschiedlichen Gestaltlösungen in einer Bibliothek hinterlegt. Da die Schnittstellenparameter der Gestaltelemente einer Gestaltungszone immer gleich sind, lassen sich die Gestaltelemente austauschen. Dadurch lassen sich mit vermindertem Aufwand topologische Änderungen durchführen [9]. In Bild 2 wird der schematische Aufbau eines GDA-Modells gezeigt. Auf der linken Seite ist eine Modellstruktur mit drei Gestaltungszonen, den Schnittstellen an den Zonengrenzen und dem Skelett dargestellt. Auf der rechten Seite sind die zur Modellstruktur passenden Gestaltelemente zu finden. Zusätzlich sind für die mittlere Gestaltungszone alternative Gestaltelemente vorgesehen.
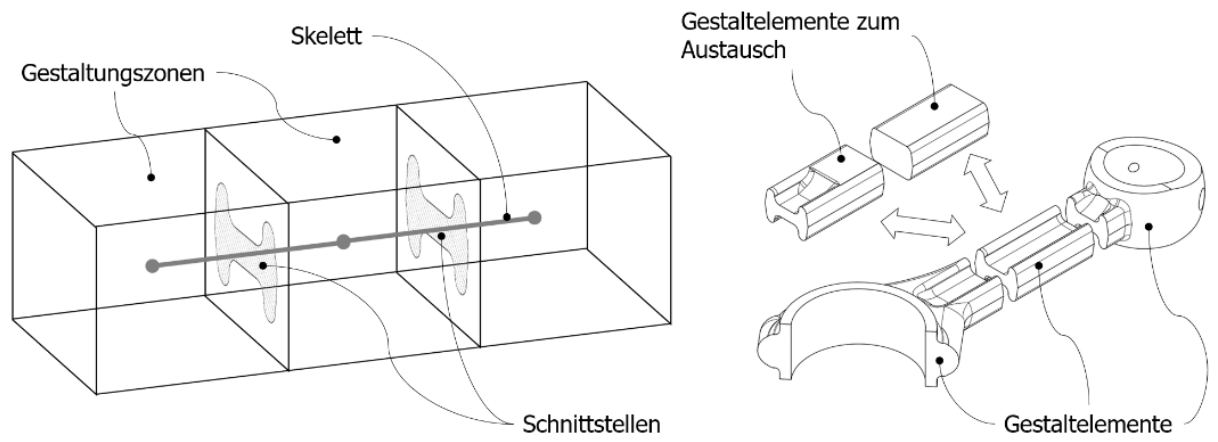

Bild 2: Schematischer Aufbau eines GDA-Modells, nach [9]

Der Vorteil ist, dass neue Bauteilvarianten, die topologische Änderungen enthalten mit reduziertem Aufwand erstellt werden können, ohne dass die Modellstruktur angepasst werden muss. Des Weiteren lässt sich das Gesamtmodell durch einige wenige Führungsparameter, die auf Baugruppenebene im Gesamtmodell definiert sind, steuern. Die Gestaltelemente passen sich dementsprechend an. Dadurch ist z.B. ein automatisierter Optimierungsprozess umgesetzt worden, bei dem durch aufeinanderfolgende Synthese- und Analyseschritte die Eigenschaften des Bauteils durch topologische und parametrische Änderungen den gestellten Anforderungen iterativ angepasst werden. Zusätzlich lassen sich in die Gestaltelemente, die zur Herstellung des Bauteils notwendigen Ferti- 
gungsrestriktionen implementieren [10]. Dadurch entstehen am Ende optimierte Bauteile mit endkonturnaher und herstellbarer Geometrie. Dabei ist zu bedenken, dass bevor ein funktionierendes Modell erstellt ist, erheblicher Aufwand zur Planung und Erstellung der Modellstruktur und der Gestaltelemente betrieben werden muss. Für jeder Gestaltungszone ist eine ausreichende Anzahl an unterschiedlichen Gestaltelementen in einer Bibliothek anzulegen, um den zur Verfügung stehenden Lösungsraum adäquat abbilden zu können. Eine mögliche Umsetzung des GDA ist in der Arbeit von Gembarski et al. zu finden. Hier ist der GDA verwendet worden, um Modelle von Strukturbauteilen (Balkenund Verbindungselemente) für den Automobilbau zu generieren [11].

\section{Modell zur Abbildung der Fertigungsstufen}

Mit dem Modell zur Abbildung der Fertigungsstufen, wird von der finalen Bauteilgeometrie aus bestimmt, ob das Bauteil mit den zur Verfügung stehenden Mitteln herstellbar ist. Dafür werden die Modelle auf Basis der Modellierungsmethode des GDA aufgebaut. Trotz der Möglichkeit für topologische Änderungen, lassen sich alle Fertigungsstufen eines Bauteils aber nicht in einem einzigen Modell unterbringen, da sich von Fertigungsstufe zu Fertigungsstufe die allgemeine Modellstruktur stark ändern kann. Aus diesem Grund wird für jede Fertigungsstufe ein separates Modell erstellt, mit einem eigenen Skelett und eigenen Gestaltelementen, die aber untereinander verknüpft sind. Die Verknüpfung der Modelle ist hierarchisch strukturiert. Im Modell der finalen Bauteilgeometrie werden die Führungsparameter definiert. Bei Änderung einer der Führungsparameter wirken sich diese erst auf das Modell der finalen Bauteilgeometrie und dann sequentiell auf die folgenden Fertigungsstufen entgegen der realen Prozesskette aus (s. Bild 3).

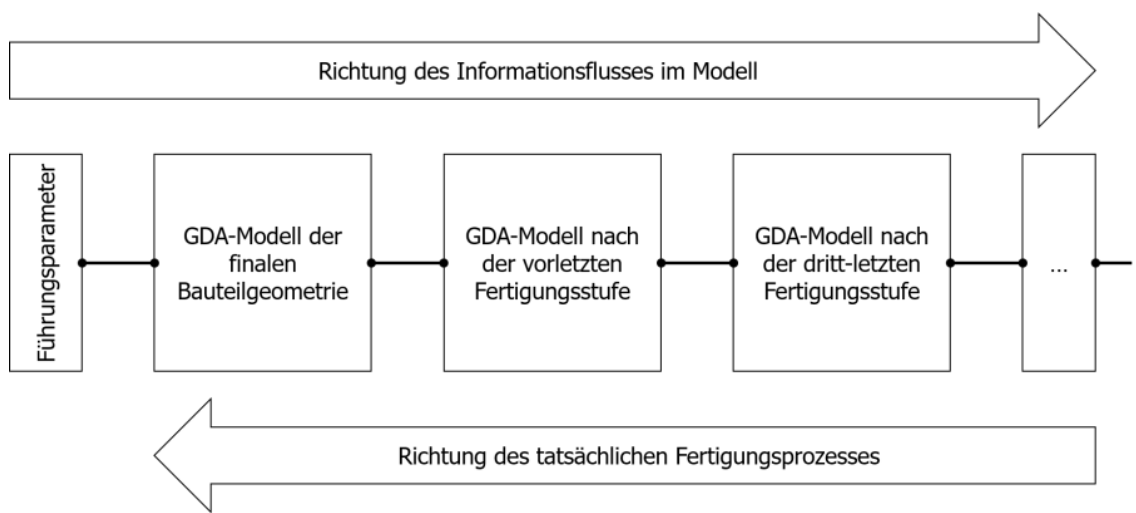

Bild 3: Informationsfluss im Modell zur Abbildung der Fertigungsstufen 
Es ist anzustreben, gleiche Modellstrukturen bei aufeinanderfolgenden Fertigungsstufen zu verwenden. Auf diese Weise gibt es die gleiche Anzahl an Gestaltungszonen und Gestaltelementen. Jedes Gestaltelement lässt sich so mit einem Gestaltelement der vorgelagerten Fertigungsstufe verknüpfen, um Geometrieinformationen konsistent zu übertragen. Bild 4 zeigt schematischen den Aufbau und die Verknüpfung der Gestaltelemente über die Fertigungsstufen. Im unteren Teil sind separat die zugehörigen Skelette dargestellt. In dem Beispiel verändert sich die Modellstruktur zwischen den beiden rechten Modellen. Die Gestaltungszone mit dem Gestaltelement 3.V ist nicht mehr vorhanden. Das ist z.B. der Fall bei einem Umformprozess, wenn eine Materialvorverteilung stattfindet. In diesem Fall wird die Geometrie von Gestaltelement 3.II durch die Gestaltelemente 2.II und 2.V definiert. Es kann unter anderem festgelegt werden, dass das Materialvolumen von 3.II exakt dem von 2.II und 2.V zusammen entsprechen muss. Auch wenn die Geometrieinformationen verknüpfter Gestaltelemente aufeinander aufbauen (z.B. GE 1.I und GE 2.I), kann ihr Aufbau grundsätzlich verschieden sein, da sich besonders die einzuhaltenden Fertigungsrestriktionen in jeder Fertigungsstufe unterscheiden.

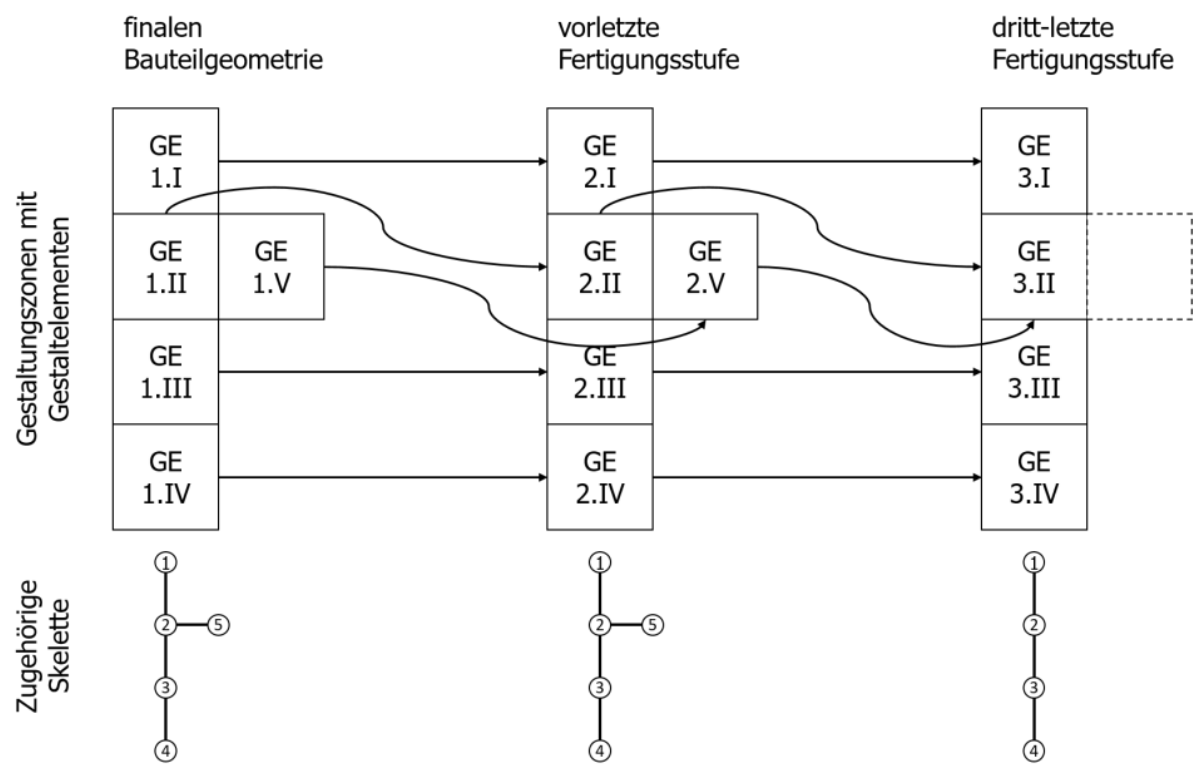

Bild 4: Schematischer Aufbau des Modells zur Abbildung der Fertigungsstufen

Im Modell der finalen Bauteilgeometrie sind grundsätzlich sowohl die meisten Abhängigkeiten innerhalb eines Gestaltelements als auch zwischen den Gestaltelementen notwendig, da hier die meisten Parameter verwendet werden, 
um die Bauteilgeometrie zu beschreiben. Der vorgestellte Ansatz nutzt die Methode zum Aufbau der Modelle nach dem GDA und die Verwendung von Skelett und Gestaltelementen. Diese Methode wird genutzt, um durch die Verknüpfung mehrerer GDA-Modelle die verschiedenen Fertigungsstufen einer Prozesskette sequenziell darstellen zu können. Außerdem wird sichtbar, welche Auswirkung eine veränderte finale Bauteilgeometrie auf die Prozesskette hat.

\section{Anwendungsbeispiel}

Als Anwendungsbeispiel wird ein rotationssymmetrischer Demonstrator aus dem SFB verwendet (s. Bild 5). Die Herstellung des hybriden Halbzeugs findet durch Reibschweißen statt, indem zwei Vollmaterialzylinder aus unterschiedlichen Werkstoffen miteinander verbunden werden. Dabei handelt es sich um ein Pressschweißverfahren, bei dem die zu verschweißenden Oberflächen aufeinander gedrückt werden. Durch eine Relativbewegung der beiden Oberflächen zueinander entsteht durch Reibung die für den Fügevorgang benötigte Schweißtemperatur [12]. Als thermomechanisches Umformverfahren wird das Voll-Vorwärts-Fleißpressen eingesetzt. Dabei wird das hybride Halbzeug mittels eines Stempels durch eine Matrize gedrückt, wodurch dessen Querschnitt gemindert wird [13]. Anschließend wird das umgeformte hybride Bauteil wärmebehandelt und spanend fertig bearbeitet. Da es bei der Wärmebehandlung keine geometrischen Veränderungen gibt, wird dieser Schritt nicht weiter betrachtet.

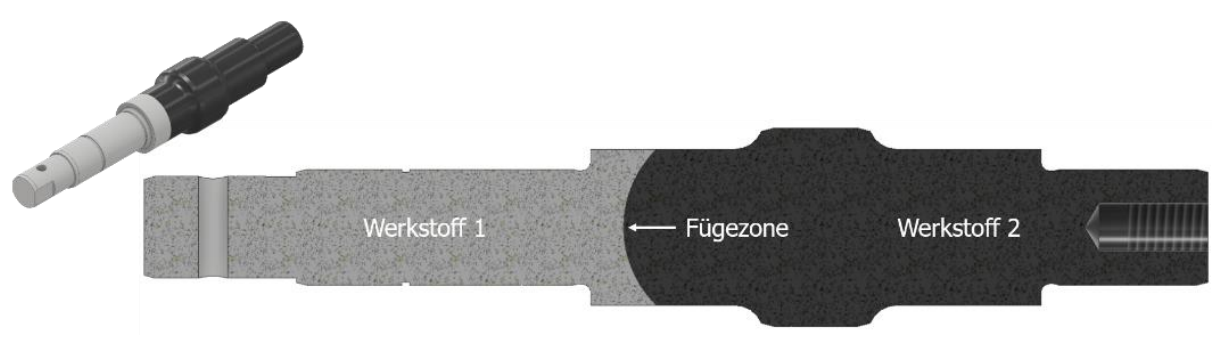

Bild 5: Modell des Demonstrators mit finaler Geometrie

Die für das Modell notwendigen Informationen bezüglich der Fertigungsrestriktionen werden durch Simulationen und reale Bauteilversuche gesammelt. Bei der Wellengeometrie hat sich ergeben, dass die für die Gestaltung zugrundeliegenden Regeln aus Tabellenwerken am besten umgesetzt werden können, wenn eine Gestaltungszone und somit ein Gestaltelement genau einen Wellenabsatz darstellt. Bild 6 zeigt eine Auswahl der durch die Bibliothek zur Verfügung gestellten Gestaltelemente für die drei betrachteten Fertigungsstufen. Die Anzahl an möglichen Gestaltelementen und deren Detailierungsgrad nimmt in 
Richtung der finalen Bauteilgeometrie zu. Das bedeutet im Umkehrschluss, dass sich viele Gestaltelemente aus den späteren Fertigungsstufen auf weniger Gestaltelemente vorheriger Fertigungsstufen beziehen lassen bzw. zu diesen reduziert werden können. Der benötigte Aufwand zur Erstellung der Gestaltelemente nimmt in Richtung der vorderen Fertigungsstufen ab.

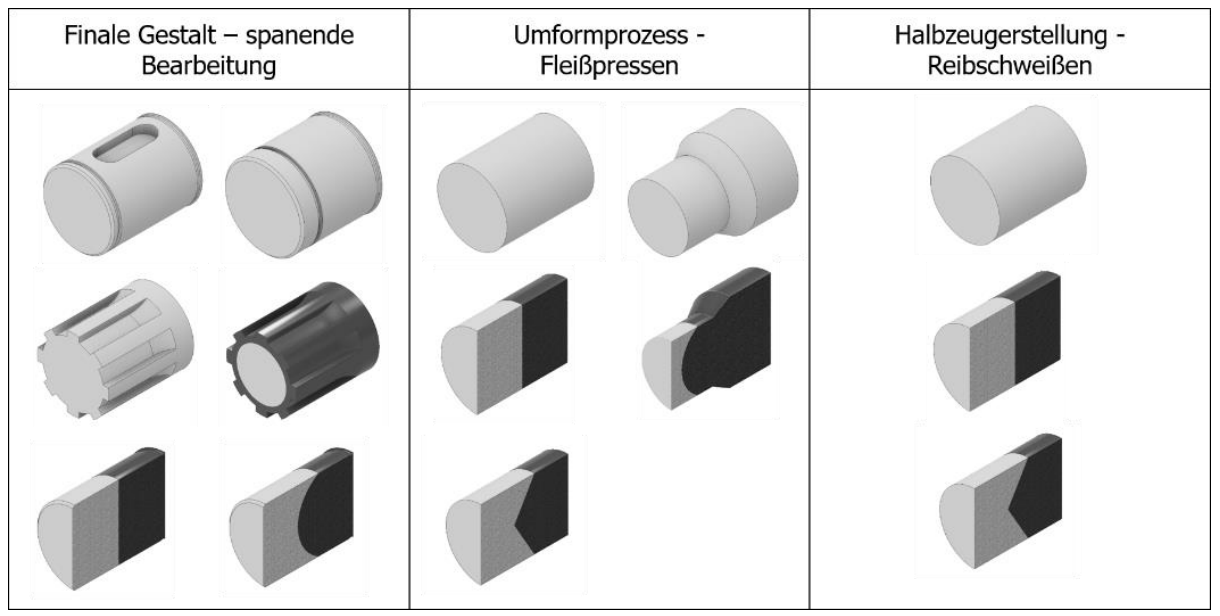

Bild 6: Auswahl verschiedene Gestaltelemente

Neben den Fertigungsstufen-übergreifenden Abhängigkeiten zwischen den Gestaltelementen, bestehen ebenso Verknüpfungen zwischen den Gestaltelementen innerhalbe eines Modells. An die benachbarten Gestaltelemente wird in der Regel der jeweilige Außendurchmesser als Parameter übergeben. Eine übergeordnete Regel sorgt dafür, dass alle Außendurchmesser von den äußeren Enden der Welle bis zum höchsten Durchmesser stets steigend ausgeführt werden, damit die benötigten Maschinenelemente auf der montiert werden können. Da es auch Gestaltmerkmale wie z.B. einen Freistich Form F oder Fügezonenkonfigurationen gibt, die sich über die Grenzen der Gestaltungszonen erstrecken, müssen auch diese berücksichtigt werden. Bild 7 zeigt am Beispiel des Freistichs Form F, dass beide Gestaltelemente über alle Informationen des Gestaltmerkmals verfügen müssen. Der ebenfalls übergebene Durchmesser $D_{1}$ dient als führende Größe bei der Freistichdimensionierung. 


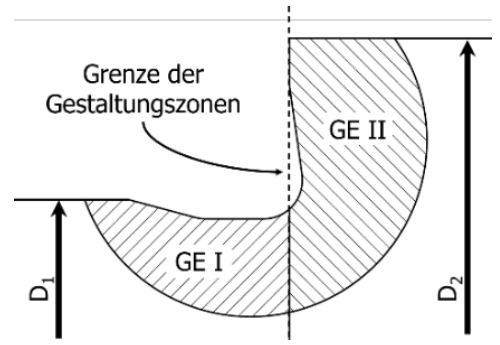

Bild 7: Beispiel für ein Gestaltmerkmal, das Gestaltungszonen-übergreifend ist; Freistich Form $\mathrm{F}$

Bild 8 zeigt das gesamte Modell zur Abbildung der Fertigungsstufen des Demonstrators. Bei rotationssymmetrischen Bauteilen ist der Vorteil, dass die Modellstruktur relativ einfach gehalten werden kann. Das Skelett bildet die Mittelachse des Bauteils und die Gestaltelemente werden hintereinander auf dieses aufgereiht.

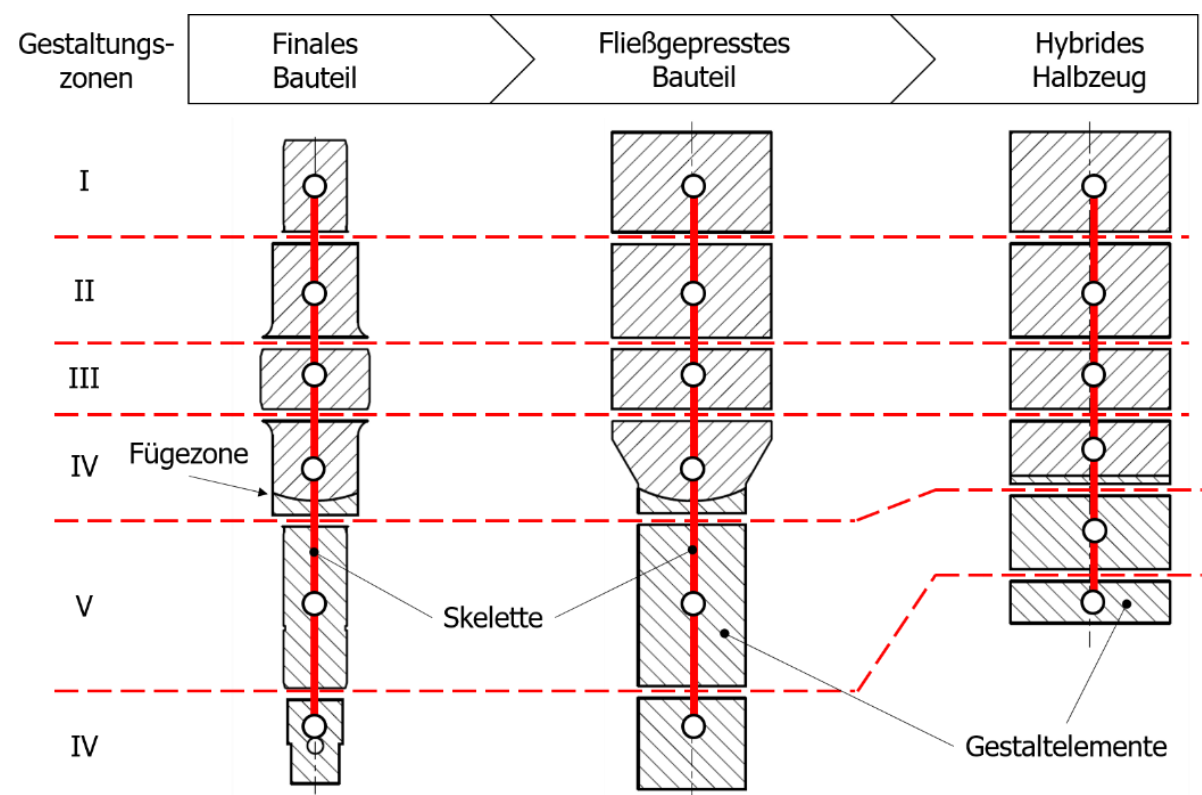

Bild 8: Darstellung der Modelle der einzelnen Fertigungsstufen inklusive Skelett und Gestaltelementen 


\section{Zusammenfassung, Diskussion und Ausblick}

Mit dem Modell zur Abbildung der Fertigungsstufen ist ein Ansatz entwickelt worden, der die Entwicklungsumgebung zur Konstruktion von Tailored-Forming-Bauteilen erweitert, indem zusätzlich, die zur Verfügung stehenden Fertigungsmöglichkeiten mitbetrachtet werden. Auf diese Weise ist es möglich, dass das zur Entwicklung hybrider Hochleistungsbauteile benötigte Konstruktionswissen verwendet werden kann, auch wenn die Konstruierenden nicht über das nötige Expertenwissen verfügen. Es lassen sich die einzelnen Fertigungsstufen eines Bauteils und die Auswirkungen einer Änderung an der finalen Bauteilgeometrie auf die Prozesskette darstellen. Der Ansatz lässt sich zudem auf weitere Technologien und Prozessketten übertragen, wenn die zur Herstellung nötigen Fertigungsrestriktionen bekannt sind.

Dabei muss berücksichtigt werden, dass spezielle anwendungsorientierte Gestaltelementbibliotheken notwendig sind, um möglichst viele verschiedene Fälle abdecken zu können. Der erforderliche Aufwand dabei lohnt sich besonders bei komplizierten Prozessketten mit vielen Fertigungsstufen, wie z.B. in Serie hergestellte Umformbauteile. Da hier der Aufwand zur Herstellung der benötigten Werkzeuge und damit verbundener möglicher Bauteilprototypen relativ hoch ist, kann mit den Modellen der Fertigungsstufen vorzeitig abgeschätzt werden, wie ein Werkzeug auszusehen hat. Sinnvoll ist der Einsatz außerdem, wenn viele ähnliche Varianten von einem Bauteil hergestellt werden, da der Entwicklungsaufwand pro Bauteil abnimmt. Das Verhältnis von Aufwand und Nutzen muss jedoch für den Einzelfall stets geklärt werden.

Neben einer Implementierung für rotationssymmetrische Bauteile, wird der Ansatz in Zukunft auch für nicht-rotationssymmetrische Bauteile umgesetzt. Die Herausforderung hierbei liegt in der Beherrschung komplizierterer Modellstrukturen, da die Anzahl an Abhängigkeiten zwischen Skelett, Gestaltelementen und den GDA-Modellen untereinander zunimmt. Zusätzlich wird die Implementierung der Fertigungsrestriktionen komplizierter als bei rotationssymmetrischen Bauteilen, da hier weniger klar definierte Auslegungsregeln durch z.B. Tabellenwerke zur Verfügung stehen.

\section{Danksagung}

Die Ergebnisse dieser Publikation sind im Rahmen des Sonderforschungsbereichs 1153 "Prozesskette zur Herstellung hybrider Hochleistungsbauteile durch Tailored Forming" innerhalb des Teilprojekts C2 "Gestaltung und Dimen- 
sionierung" entstanden. Die Autoren danken der Deutschen Forschungsgemeinschaft (DFG) für die finanzielle und organisatorische Unterstützung des Projektes.

\section{Literatur}

[1] Behrens, B.-A., et al.: "Simulation-Aided Process Chain Design for the Manufacturing of Hybrid Shafts", HTM Journal of Heat Treatment and Materials, Vol. 74(2), 2019, S. $115-135$

[2] Behrens, B.-A., et al.: „Angepasste Erwärmungs- und Umformverfahren für hybride Massivbauteile", 23. Sächsische Fachtagung Umformtechnik (SFU), Dresden, 2016

[3] Brockmöller, T., et al.: "Design Catalogue in a CAE Environment for the Illustration of Tailored Forming", In: Scharff, P., et al. (Hrsg.): 59th $\mathrm{Il}-$ menau Scientific Colloquium - Engineering for a Changing World, ilmedia, Ilmenau, 2017, 5.02

[4] Siqueira, R., Mozgova, I., Lachmayer, R.: "An Interfacial Zone Evolutionary Optimization Method with Manufacturing Constraints for Hybrid Components", Journal of Computational Design and Engineering, Vol. 6, 2018, S. 387-397

[5] Siqueira, R., et al.: "An adapted case-based reasoning system for design and manufacturing of tailored forming multi-material components", International Journal on Interactive Design and Manufacturing, 2019, S. 110

[6] Vajna, S.; et al.: "CAx für Ingenieure", 2. Auflage, Springer-Verlag, Berlin, Heidelberg, 2009

[7] Chapman, C., Pinfold, M.: "The application of a knowledge based engineering approach to the rapid design and analysis of an automotive structure", Advances in Engineering Software, Vol. 32, 2001, S. 903-912

[8] Hirz, M.; et al.: "Integrated Computer-Aided Design in Automotive Development", Springer Verlag, Heidelberg, 2013

[9] Sauthoff, B.: "Generative parametrische Modellierung von Strukturkomponenten für die technische Vererbung", TEWISS - Technik und Wissen GmbH Verlag, Garbsen, 2017 
[10] Gembarski, P.; et al.: "Operationalization of Manufacturing Restrictions for CAD and KBE-Systems", In: Štorga, D.; et al. (Hrsg.): Proceedings of the DESIGN 2016 - 14th International Design Conference - Vol. 3.: Design Support Tools, The Design Society, Glasgow, 2016, S. 621-630

[11] Gembarski, P.; Li, H.; Lachmayer, R.: "Template-based modelling of structural components", International Journal of Mechanical Engineering and Robotics Research, 6(5), 2017, S. 336-342

[12] Dilthey, U.: ,Schweißtechnische Fertigungsverfahren 1", Springer-Verlag, Berlin, Heidelberg, 2006

[13] Doege, E.; Behrens, B.-A.: „Handbuch Umformtechnik”, Springer-Verlag, Berlin, Heidelberg, 2010 